\title{
Comparison of Real-World Treatment Patterns Among Biologic-Experienced Patients with Psoriasis Treated with Ixekizumab or Secukinumab Over 18 Months
}

\author{
Andrew Blauvelt $\cdot$ Nianwen Shi $\cdot$ Russel Burge $\cdot$ Najwa Somani $\cdot$ \\ Terri L. Ridenour • Baojin Zhu • Bilal Atiya • Carolyn R. Lew • \\ Nicole M. Zimmerman · Mwangi J. Murage
}

Received: July 21, 2021 / Accepted: October 4, 2021 / Published online: October 15, 2021

(C) The Author(s) 2021

\begin{abstract}
Introduction: Real-world data comparing effectiveness of ixekizumab (IXE) and secukinumab (SEC) among biologic-experienced patients are limited. This study compared treatment patterns over 18 months among biologic-experienced patients with psoriasis receiving IXE or SEC in the USA.

Methods: A retrospective observational study using administrative claims data from IBM $^{\circledR}$ Watson Health MarketScan ${ }^{\circledR}$ Research Databases included adult patients with $\geq 1$ inpatient or $\geq 2$ non-diagnostic ( $\geq 30$ days apart) outpatient claim/s with diagnosis of psoriasis between March 1, 2015 and October 31, 2019, and $\geq 1$ claim/s for index drugs, IXE or SEC, between March 1, 2016 and October 31, 2019. Patients had to have $\geq 1 \mathrm{claim} / \mathrm{s}$ for biologics indicated for psoriasis in the 6-month pre-period. During the 18-month follow-up, treatment adherence
\end{abstract}

\footnotetext{
A. Blauvelt

Oregon Medical Research Center, Portland, OR, USA

N. Shi · C. R. Lew · N. M. Zimmerman

IBM Watson Health, Cambridge, MA, USA

R. Burge · N. Somani · T. L. Ridenour · B. Zhu

B. Atiya · M. J. Murage ( $\square)$

Eli Lilly and Company, Indianapolis, IN, USA

e-mail: murage_mwangi_james@lilly.com

R. Burge

University of Cincinnati, Cincinnati, OH, USA
}

(proportion of days covered [PDC]), high adherence ( $\mathrm{PDC} \geq 80 \%$ ), persistence, discontinuation, reinitiation, and switching were assessed. To address cohort imbalances, inverse probability of treatment weighting was employed. Logistic regression was used to estimate odds ratio for high adherence. Cox proportional hazard models were used to estimate hazard ratio for non-persistence, discontinuation, and switching.

Results: Overall, 411 IXE and 780 SEC users were included. After weighting, IXE users had significantly higher rate of high treatment adherence $(42 \%$ vs. $35 \%, p=0.019)$, higher persistence rate $(44.9 \%$ vs. $36.9 \%, p=0.007)$, lower discontinuation rate $(48.4 \%$ vs. $56.0 \%$, $p=0.012)$, and lower switching rate $(26.6 \%$ vs. $34.0 \%, p=0.009)$ compared with SEC users. After multivariable adjustment, compared with SEC, IXE use was associated with $36 \%$ higher odds of high treatment adherence (OR 1.36, 95\% CI 1.05-1.74), 20\% lower risk of treatment non-persistence (HR 0.80, 95\% CI 0.68-0.93), $19 \%$ lower risk of discontinuation (HR 0.81, 95\% CI 0.68-0.96), and 25\% lower risk of switching (HR 0.75, 95\% CI 0.60-0.93).

Conclusion: This study suggests that IXE treatment is associated with significantly higher adherence rates and significantly lower nonpersistence, discontinuation, and switching compared with SEC treatment. 
Keywords: Biologic-experienced; Ixekizumab; Psoriasis; Real-world evidence; Secukinumab; Treatment adherence; Treatment discontinuation; Treatment patterns; Treatment persistence; Treatment switching

\section{Key Points}

\section{Why carry out this study?}

Efficacy of biologics among prior biologicexperienced patients with psoriasis may be lower because of development of antibodies against the drug.

In the clinical setting, newer biologics such as ixekizumab (IXE) and secukinumab (SEC) have demonstrated similar efficacy among patients with or without prior biologic treatment; however, head-to-head comparisons between two IL-17A inhibitors are lacking.

We carried out this retrospective study based on real-world claims data to compare treatment patterns of IXE and SEC among biologic-experienced patients with psoriasis.

\section{What was learned from the study?}

Among patients with psoriasis who were previously treated with biologics, IXE users demonstrated higher rates of adherence and persistence, and lower rates of discontinuation and switching compared with SEC users over 18-month follow-up.

These results may help healthcare practitioners in the selection of biologic treatment for biologic-experienced patients with psoriasis.

\section{INTRODUCTION}

Data from clinical trials and real-world settings have demonstrated that biologics are effective in treating patients with moderate-to-severe psoriasis [1-6]. The Joint American Academy of Dermatology and National Psoriasis Foundation guidelines recommend biologics for the treatment of moderate-to-severe psoriasis [7]. Continuous treatment with biologics is recommended to achieve effective disease management in patients with psoriasis; however, in real-world settings, about $18-46 \%$ of patients discontinue their biologic therapy within the first year of treatment. This interruption leads to symptom recurrence [8-10]. Although prior biologic use is reported to be associated with poor anticipated biologic treatment efficacy, the newer interleukin (IL)-17A inhibitors (ixekizumab [IXE], secukinumab [SEC], and brodalumab) have demonstrated high efficacy among biologic-experienced patients [11-13].

Clinical trials indirectly indicate that IXE and SEC show comparable efficacy irrespective of their prior biologic use in patients with psoriasis; however, head-to-head comparisons are lacking in clinical trials and limited in realworld settings [6, 14-16]. Here, we compared persistence, adherence, discontinuation, reinitiation, and switching over 18 months of follow-up among biologic-experienced patients with psoriasis treated with either IXE or SEC in real-world settings.

\section{METHODS}

\section{Data Sources}

A retrospective observational study design was used to compare IXE and SEC treatment patterns among biologic experienced patients with psoriasis using health insurance claims from IBM $^{\circledR}$ Watson Health MarketScan ${ }^{\circledR}$ Research Databases.

The MarketScan ${ }^{\circledR}$ databases comprised the (1) Commercial Claims and Encounter database that contains information on inpatient and outpatient medical and outpatient pharmacy experiences of employees and their dependents covered under fee-for-service with fully and partially capitated health plans; (2) Medicare supplemental database, which has the same information for individuals with Medicare 
supplemental insurance paid by employers; and (3) Early View database that captures similar information incurred as late as approximately 45 days before data release.

This study was conducted in accordance with the Good Practices for Real-World Data Studies of Treatment and/or Comparative Effectiveness [17]. All study data were accessed with protocol compliant with the US patient confidentiality requirements, including the Health Insurance Portability and Accountability Act (HIPAA) of 1996 regulations. As all databases used in the study are fully de-identified and compliant with the HIPAA, this study was exempt from institutional review board approval.

\section{Study Population}

Eligible patients included adults (at least 18 years old) with at least one inpatient or at least two non-diagnostic outpatient claims (at least 30 days apart) with a diagnosis of psoriasis (International Classification of Diseases, Ninth Revision, Clinical Modification [ICD-9-CM] diagnosis code $696.1 \mathrm{x}$ or International Classification of Diseases, Tenth Revision, Clinical Modification [ICD-10-CM] diagnosis codes L40.0-L40.4 or L40.8-L40.9) between March 1, 2015 and October 31, 2019. Patients were required to have at least one claim for the index drug, IXE or SEC, between March 1, 2016 and October 31, 2019, with a diagnosis of psoriasis before or coinciding with the index drug. Patients were also required to have at least 6 months pre- and at least 18 months post-index continuous enrollment for medical and pharmacy benefits. Biologic-experienced patients were identified as patients with at least one pre-index claim of following biologic drugs indicated for psoriasis: certolizumab pegol, etanercept, adalimumab, infliximab, brodalumab, ustekinumab, guselkumab, secukinumab, and ixekizumab. Patients were excluded if they had evidence of other indications for IXE or SEC (psoriatic arthritis or ankylosing spondylitis) during the pre-index period, or if they had the index medication on hand within 90 days before the index date. Index date was the date of the first IXE or SEC claim and patients were assigned to IXE or SEC group based on the index drug.

\section{Dosing}

For ixekizumab, the dosing schedule consisted of initial $160 \mathrm{mg}$ at week 0 , followed by $80 \mathrm{mg}$ at weeks $2,4,6,8,10$, and 12 , and then $80 \mathrm{mg}$ every 4 weeks. For secukinumab, the dosing schedule included $300 \mathrm{mg}$ at weeks $0,1,2,3$, and 4 , and then $300 \mathrm{mg}$ every 4 weeks.

\section{Outcomes}

Treatment pattern outcomes during the 18-month follow-up period included treatment adherence, persistence, discontinuation, reinitiation, and switching. These were defined as follows:

- Adherence was measured as the proportion of days covered (PDC), calculated by dividing the total number of days of index drug supply in the follow-up period by the total number of days in the follow-up period. When dates of two index drug claims overlapped, the start date of the second index drug claim was adjusted to the date after the last day supply for the first index drug claim. The number of days of supply from the last claim beyond the end of follow-up was truncated. High adherence was defined as PDC $\geq 80 \%$.

$$
\text { PDC }=\frac{\text { Total number of days of index drug supply during the follow-up period }}{\text { Total number of days in follow-up period }}
$$


- Treatment persistence was defined as having a gap of less than 60 days between the last day of supply of the prior prescription and the next refill. The end date of persistence was set by the last day supply of the prescription before the 60-day gap. If a patient's last days' supply of their index medication class occurred with less than 60 days to the end of the follow-up period, persistence was calculated using the last days' supply and was not extrapolated beyond.

- Treatment discontinuation was defined as no refill of the index drug within 90 days after the previous days' supply was exhausted. The date of discontinuation was set as the last day supply of index drug before reaching the 90-day gap.

- Treatment reinitiation was measured among the subset of patients who discontinued the index therapy and was defined as having at least one claim of the index drug after the discontinuation date.

- Treatment was considered switched if a different biologic indicated for psoriasis (adalimumab, brodalumab, certolizumab pegol, etanercept, guselkumab, infliximab, IXE, SEC, ustekinumab, tildrakizumab, risankizumab) was prescribed and the date of the first new biologic claim set the switching date.

\section{Covariates}

Age, sex, primary payer, health plan type, and geographic region were measured on the index date. Baseline clinical characteristics included Deyo-Charlson Comorbidity Index, comorbid conditions (diabetes, anxiety, depression, hypertension, hyperlipidemia, coronary heart disease, peripheral vascular disease, obesity, sleep apnea, osteoarthritis, and other autoimmune disorders), and all-cause and psoriasis-related healthcare costs. Baseline medications used for psoriasis included biologics, systemic agents/targeted oral therapies (apremilast, acitretin, systemic steroids, other systemic agents [cyclosporine, methotrexate, azathioprine, hydroxyurea, isotretinoin, leflunomide, methoxsalen, mycophenolate mofetil, sulfasalazine, or thioguanine]), topical treatment, and phototherapy.

\section{Statistical Analyses}

Categorical variables were summarized by the count and proportion of patients in each category. Continuous variables were presented as mean and standard deviation (SD). To address cohort imbalance, inverse probability of treatment weighting (IPTW) was employed using a logistic regression model with IXE versus SEC as a dependent variable. Demographics, baseline clinical characteristics, pre-period use of systemic agents/targeted oral therapies/topical agents for psoriasis, pre-period phototherapy, $\log$ (pre-period psoriasis-related costs), and preperiod comorbidity-related costs were included in the models. Cohorts were balanced if a standardized difference of $10 \%$ or less was attained. Statistical tests for significance were conducted using a weighted chi-square test for categorical variables and weighted $t$ test for continuous variables. Kaplan-Meier (KM) estimation and log-rank test were employed using the weighted samples. Cox proportional hazards models were used to estimate the hazard ratio (HR) for persistence, discontinuation, and switching. Logistic regression was performed to estimate the odds ratio (OR) of high adherence measured by PDC $\geq 80 \%$. Multivariable models were adjusted for the same list of covariates used in the IPTW model. HR and OR were presented along with 95\% confidence intervals (CI). A $p$ value of 0.05 was set a priori as the threshold for statistical significance.

Descriptive analyses were conducted using WPS version 4.2 (World Programming, UK); IPTW and multivariable analyses were conducted using $\mathrm{R}$ version 3.6.3 (R Foundation for Statistical Computing, Vienna, Austria).

\section{RESULTS}

A total of 411 biologic-experienced IXE users and 780 SEC users were included in the study (Fig. 1). Baseline demographics and clinical characteristics before and after weighting are presented in Table 1. Mean age of patients for 
Patients with $\geq 1$ inpatient or $\geq 2$ outpatient nondiagnostic claims ( $\geq 30$ days apart) for psoriasis between March 1, 2015 and October 31, 2019, occurring on or after first $\mathrm{PsO}$ diagnosis date

$$
\begin{array}{cc}
\text { IXE, } & \text { SEC, } \\
n=261,539 & \mathrm{n}=261,539
\end{array}
$$

\begin{tabular}{|c|c|c|}
\hline $\begin{array}{l}\text { AND } \geq 1 \text { claim for IXE or SEC between } \\
\text { March } 01,2016 \text { and October } 31,2019\end{array}$ & $\begin{array}{c}\text { IXE, } \\
\mathrm{n}=14,604\end{array}$ & $\begin{array}{c}\mathrm{SEC}, \\
\mathrm{n}=14,604\end{array}$ \\
\hline $\begin{array}{l}\text { AND index drug as IXE or SEC, } \\
\text { AND } \geq 18 \text { years old on index date }\end{array}$ & $\begin{array}{c}\mathrm{IXE}, \\
\mathrm{n}=4,080\end{array}$ & $\begin{array}{c}\mathrm{SEC}, \\
\mathrm{n}=10,512\end{array}$ \\
\hline $\begin{array}{l}\text { AND continuous enrollment with medical and } \\
\text { pharmacy benefits and no diagnosis of other } \\
\text { indications for index drug for } \geq 6 \text { months pre- } \\
\text { period }\end{array}$ & $\begin{array}{l}\mathrm{IXE} \\
\mathrm{n}=2,510\end{array}$ & $\begin{array}{c}\mathrm{SEC} \\
\mathrm{n}=4,692\end{array}$ \\
\hline $\begin{array}{c}\text { AND no pharmacy claim for index drug } 90 \\
\text { days before index date }\end{array}$ & $\begin{array}{c}\text { IXE, } \\
n=2,470\end{array}$ & $\begin{array}{l}\mathrm{SEC} \\
\mathrm{n}=3,975\end{array}$ \\
\hline $\begin{array}{c}\text { AND post-index } \geq 18 \text { months continuous } \\
\text { enrollment with medical and pharmacy } \\
\text { benefits }\end{array}$ & $\begin{array}{c}\mathrm{IXE} \text {, } \\
\mathrm{n}=747\end{array}$ & $\begin{array}{c}\mathrm{SEC} \\
\mathrm{n}=1,508\end{array}$ \\
\hline $\begin{array}{l}\text { AND with a pre-index biologic use for } \\
\text { psoriasis (biologic-experience) }\end{array}$ & $\begin{array}{c}\mathrm{IXE}, \\
\mathrm{n}=411\end{array}$ & $\begin{array}{c}\mathrm{SEC}, \\
\mathrm{n}=780\end{array}$ \\
\hline
\end{tabular}

Fig. 1 Patient attrition flow chart. $I X E$ ixekizumab, $S E C$ secukinumab

both treatment groups was 49 years. Before weighting, the proportion of men was $56.9 \%$ for the IXE group and $52.9 \%$ for the SEC group. Most patients were enrolled in commercial payer coverage (IXE vs. SEC, 95.9\% vs. 94.7\%), and more than half of patients had either exclusive provider organization or preferred provider organization insurance plans (IXE vs. SEC, $60.3 \%$ vs. $57.6 \%$ ). Nearly half of the patients in both treatment groups were from the southern USA (IXE vs. SEC, $48.7 \%$ vs. $48.3 \%)$.

Overall, baseline and demographic characteristics were balanced after weighting. Mean
(SD) Deyo-Charlson Comorbidity Index scores were similar among IXE (0.4 [0.8]) and SEC (0.4 [0.9]) users. Common comorbid conditions among IXE versus SEC groups at baseline were hypertension $(26.8 \%$ vs. $27.4 \%)$, hyperlipidemia $(21.6 \%$ vs. $21.9 \%)$, obesity $(19.0 \%$ vs. $18.9 \%)$, diabetes $(15.3 \%$ vs. $15.4 \%)$, and sleep apnea (10.3\% vs. $10.4 \%)$.

\section{Adherence and Persistence}

During the 18-month follow-up period, mean (SD) PDC among IXE users was significantly 
Table 1 Demographics and baseline characteristics, before and after weighting, ixekizumab or secukinumab

\begin{tabular}{|c|c|c|c|c|c|c|}
\hline \multirow[t]{2}{*}{ Variables } & \multicolumn{3}{|c|}{ Before weighting } & \multicolumn{3}{|c|}{ After weighting } \\
\hline & $\begin{array}{l}\text { IXE } \\
(n=411)\end{array}$ & $\begin{array}{l}\text { SEC } \\
(n=780)\end{array}$ & $\begin{array}{l}\text { Std } \\
\text { Diff }^{a}\end{array}$ & IXE & SEC & $\begin{array}{l}\text { Std } \\
\text { diff }\end{array}$ \\
\hline Age, years, mean $(\mathrm{SD})$ & $48.8(10.8)$ & $48.5(11.5)$ & 2.0 & $48.5(11.0)$ & $48.6(11.3)$ & 0.9 \\
\hline Male (\%) & 56.9 & 52.9 & 8.0 & 54.2 & 54.4 & 0.3 \\
\hline Pre-index DCCI, mean (SD) & $0.3(0.7)$ & $0.4(0.9)$ & 9.0 & $0.4(0.8)$ & $0.4(0.9)$ & 3.3 \\
\hline Commercial primary payer (\%) & 95.9 & 94.7 & 5.3 & 95.4 & 95.2 & 1.1 \\
\hline Insurance plan type (\%) & & & 28.9 & & & 13.0 \\
\hline $\mathrm{EPO} / \mathrm{PPO}$ & 60.3 & 57.6 & & 59.0 & 57.9 & \\
\hline CDHP, HDHP & 25.1 & 18.2 & & 21.0 & 20.8 & \\
\hline $\mathrm{HMO}$ & 7.1 & 12.8 & & 11.0 & 10.8 & \\
\hline POS/POS with capitation & 4.1 & 5.3 & & 4.7 & 4.8 & \\
\hline Comprehensive/indemnity & 3.2 & 4.7 & & 4.1 & 4.2 & \\
\hline Geographic region (USA) (\%) & & & 17.5 & & & 7.5 \\
\hline South & 48.7 & 48.3 & & 49.0 & 48.3 & \\
\hline North Central & 27.0 & 21.8 & & 23.8 & 23.8 & \\
\hline Northeast & 14.6 & 16.0 & & 15.6 & 15.5 & \\
\hline West & 9.7 & 13.6 & & 11.6 & 12.2 & \\
\hline Unknown & 0.0 & 0.3 & & 0.0 & 0.3 & \\
\hline \multicolumn{7}{|l|}{ Comorbid conditions (\%) } \\
\hline Hypertension & 26.0 & 28.2 & 4.9 & 26.8 & 27.4 & 1.3 \\
\hline Hyperlipidemia & 22.1 & 21.8 & 0.8 & 21.6 & 21.9 & 0.7 \\
\hline Obesity & 19.7 & 18.3 & 3.5 & 19.0 & 18.9 & 0.4 \\
\hline Diabetes & 15.8 & 15.0 & 2.3 & 15.3 & 15.4 & 0.3 \\
\hline Sleep apnea & 10.0 & 10.6 & 2.2 & 10.3 & 10.4 & 0.4 \\
\hline Anxiety & 8.5 & 9.1 & 2.1 & 8.8 & 8.8 & 0.2 \\
\hline Depression & 7.8 & 7.4 & 1.3 & 7.6 & 7.6 & 0.1 \\
\hline Osteoarthritis & 6.6 & 8.2 & 6.3 & 7.4 & 7.6 & 0.8 \\
\hline \multicolumn{7}{|l|}{ Pre-index period treatment } \\
\hline Any topical agent (\%) & 59.4 & 59.9 & 1.0 & 59.6 & 59.7 & 0.1 \\
\hline Any systemic/targeted oral agent (\%) & 36.3 & 34.5 & 3.7 & 34.9 & 35.1 & 0.4 \\
\hline Phototherapy or laser therapy (\%) & 1.7 & 1.5 & NA & 1.5 & 1.6 & NA \\
\hline $\begin{array}{l}\text { Number of unique biologics used in pre-index } \\
\text { period (mean, SD) }\end{array}$ & $1.0(0.1)^{\mathrm{b}}$ & $1.0(0.2)^{\mathrm{b}}$ & 4.0 & $1.0(15.2)^{\mathrm{b}}$ & $1.0(16.5)^{b}$ & 2.7 \\
\hline
\end{tabular}


Table 1 continued

\begin{tabular}{|c|c|c|c|c|c|c|}
\hline \multirow[t]{2}{*}{ Variables } & \multicolumn{3}{|c|}{ Before weighting } & \multicolumn{3}{|c|}{ After weighting } \\
\hline & $\begin{array}{l}\text { IXE } \\
(n=411)\end{array}$ & $\begin{array}{l}\text { SEC } \\
(n=780)\end{array}$ & $\begin{array}{l}\text { Std } \\
\text { Diff }\end{array}$ & $\overline{\mathrm{IXE}}$ & SEC & $\begin{array}{l}\text { Std } \\
\text { diff }^{\mathrm{a}}\end{array}$ \\
\hline \multicolumn{7}{|c|}{ Pre-index healthcare costs, in USD, mean $(S D)^{c}$} \\
\hline All-cause & $\begin{array}{l}\$ 4695 \\
\quad(\$ 2875)\end{array}$ & $\begin{array}{l}\$ 4718 \\
\quad(\$ 3660)\end{array}$ & 0.7 & $\begin{array}{l}\$ 4644 \\
\quad(\$ 2857)\end{array}$ & $\begin{array}{l}\$ 4725 \\
\quad(\$ 3576)\end{array}$ & 2.5 \\
\hline Psoriasis-specific & $\begin{array}{l}\$ 3895 \\
\quad(\$ 2021)\end{array}$ & $\begin{array}{l}\$ 3810 \\
\quad \$ 1946)\end{array}$ & 4.3 & $\begin{array}{l}\$ 3850 \\
\quad \$ 2049)\end{array}$ & $\begin{array}{l}\$ 3842 \\
\quad \$ 1955)\end{array}$ & 0.4 \\
\hline
\end{tabular}

$C D H P$ consumer-driven health plan, DCCI Deyo-Charlson Comorbidity Index, EPO exclusive provider organization, $H D H P$ high deductible health plan, $H M O$ health maintenance organization, $I X E$ ixekizumab, POS point of service, PPO preferred provider organization, $S D$ standard deviation, SEC secukinumab, Std Diff standardized difference, USD US dollar ${ }^{a}$ A standardized difference of $<10 \%$ is considered well balanced

b Value reported as mean $(\mathrm{SD})$

${ }^{c}$ Reported as per patient per month

higher compared with SEC users $(0.63$ [0.31] vs. $0.59[0.30], p=0.046)$. A significantly higher proportion of IXE users were highly treatment adherent $(\mathrm{PDC} \geq 80 \%)$ than SEC users $(42.0 \%$ vs. $35.0 \%, p=0.019)$. A significantly higher proportion of IXE users had persistent treatment through 18 months $(44.9 \%$ vs. $36.9 \%$, $p=0.007)$ than SEC users. In addition, IXE users had a significantly higher mean (SD) number of days on persistent treatment than SEC users (356 [196] vs. 325 [196], $p=0.008$ ) (Table 2).

KM estimation showed that the probability of persistence was significantly higher for IXE users compared with SEC users $(p=0.007)$ (Fig. 2a). The median time to $50 \%$ non-persistence was 416 days (95\% CI 361-not available) for the IXE group versus 319 days (95\% CI 285-350) for the SEC group. In multivariable analysis, IXE users had $36 \%$ higher odds of being highly treatment-adherent (OR 1.36, 95\% CI 1.05-1.74) and had 20\% lower risk of non-persistence (HR 0.80, 95\% CI 0.68-0.93) compared with SEC users (Fig. 3).

\section{Discontinuation and Reinitiation}

A significantly lower proportion of IXE users discontinued treatment $(48.4 \%$ vs. $56.0 \%$, $p=0.018$ ) compared with SEC users. Mean (SD) number of days to discontinuation was similar between the two treatment groups (197 [123] vs. 200 [118], $p=0.764$ ) (Table 2). Based on KM estimation, the probability of discontinuation was significantly lower for IXE users compared to SEC users $(p=0.018)$. Median time to discontinuation among SEC users was 364 days (95\% CI 325-418). In IXE users, median time to discontinuation was not available because less than $50 \%$ of IXE users discontinued by the end of 18-month follow-up period (Fig. 2b). The proportion of patients reinitiating after discontinuation (IXE vs. SEC, $9.2 \%$ vs. $10.5 \%$, $p=0.473$ ) and mean (SD) number of days from discontinuation to reinitiation (IXE vs. SEC, 164 [59] vs. 173 [77], $p=0.564$ ) were similar between the treatment groups (Table 2). In multivariable analysis, IXE users had a 19\% lower risk of discontinuation compared with SEC users (HR 0.81, 95\% CI 0.68-0.96) (Fig. 3).

\section{Switching}

IXE users had a significantly lower rate of switching from index treatment than SEC users (26.6\% vs. $34.0 \% ; p=0.009$ ). The top three new biologics among IXE users were SEC (9.2\%), guselkumab (6.5\%), and ustekinumab (4.2\%); among SEC users, these were IXE (13.6\%), guselkumab (6.0\%), and adalimumab (6.0\%). The mean (SD) number of days to first switch 
Table 2 Post-weighting persistence, discontinuation, reinitiation, and switching over 18-month follow-up, ixekizumab or secukinumab

\begin{tabular}{|c|c|c|c|}
\hline & IXE & SEC & $p$ value \\
\hline \multicolumn{4}{|l|}{ Adherence } \\
\hline PDC, mean (SD) & $0.63(0.31)$ & $0.59(0.30)$ & 0.046 \\
\hline Patients with PDC $\geq 80 \%$ (\%) & 42.0 & 35.0 & 0.019 \\
\hline \multicolumn{4}{|l|}{ Persistence (60-day gap) } \\
\hline Patients who were persistent (\%) & 44.9 & 36.9 & 0.007 \\
\hline Days on persistent treatment, mean (SD) & $356(196)$ & $325(196)$ & 0.008 \\
\hline \multicolumn{4}{|l|}{ Discontinuation (90-day gap) } \\
\hline Patients who discontinued treatment (\%) & 48.4 & 56.0 & 0.012 \\
\hline Days to discontinuation, mean (SD) & $197(123)$ & $200(118)$ & 0.764 \\
\hline \multicolumn{4}{|l|}{ Reinitiation after discontinuation } \\
\hline Patients who reinitiated treatment (\%) & 9.2 & 10.5 & 0.473 \\
\hline Days from discontinuation to reinitiation, mean (SD) & $164(59)$ & $173(77)$ & 0.564 \\
\hline \multicolumn{4}{|l|}{ Switching } \\
\hline Patients who switched from index treatment (\%) & 26.6 & 34.0 & 0.009 \\
\hline Days to first switch, mean (SD) & $269(141)$ & $271(131)$ & 0.921 \\
\hline \multicolumn{4}{|l|}{ First switch from index drug to (\%) } \\
\hline IXE & NA & 13.6 & NA \\
\hline SEC & 9.2 & NA & NA \\
\hline Guselkumab & 6.5 & 6.0 & 0.755 \\
\hline Ustekinumab & 4.2 & 4.6 & 0.765 \\
\hline Adalimumab & 2.9 & 6.0 & 0.021 \\
\hline Etanercept & 2.4 & 2.0 & NA \\
\hline
\end{tabular}

$p$ values were obtained using weighted $t$ tests for continuous variables and weighted chi-square tests for binary or categorical variables

$p$ value was unavailable because fewer than 30 patients switched to etanercept in both study cohorts

$I X E$ ixekizumab, $N A$ not available, $P D C$ proportion of days covered, $S D$ standard deviation, $S E C$ secukinumab

was similar between the two groups (269 [141] vs. 271 [131], $p=0.921$ ) (Table 2). The probability of switching was significantly lower for IXE users compared to SEC users $(p=0.011)$. Median time to $50 \%$ switching was not available because by the end of 18-month follow-up period, less than $50 \%$ of patients switched in both groups. (Fig. 2c). In the multivariate analysis, IXE users had a $25 \%$ lower risk of switching
Fig. 2 Probability of treatment persistence, discontinuation, and switching over 18 months follow-up, patients with psoriasis receiving ixekizumab or secukinumab. a Probability of treatment persistence (60-day treatment gap). b Probability of treatment discontinuation (90-day treatment gap). c Probability of treatment switching. IXE ixekizumab, SEC secukinumab. Note: Probability of switching for both IXE and SEC on day 90 was 3\% 

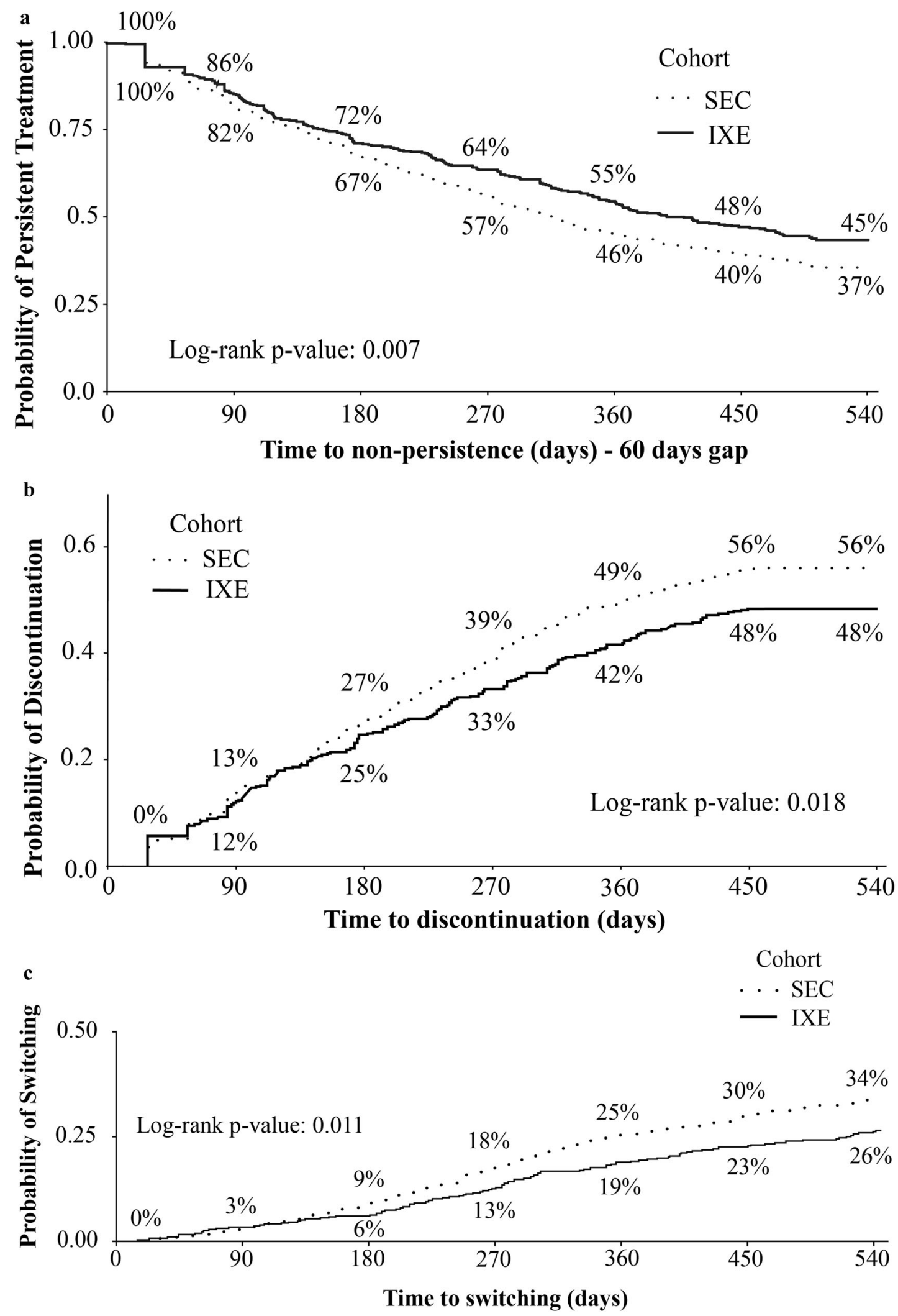


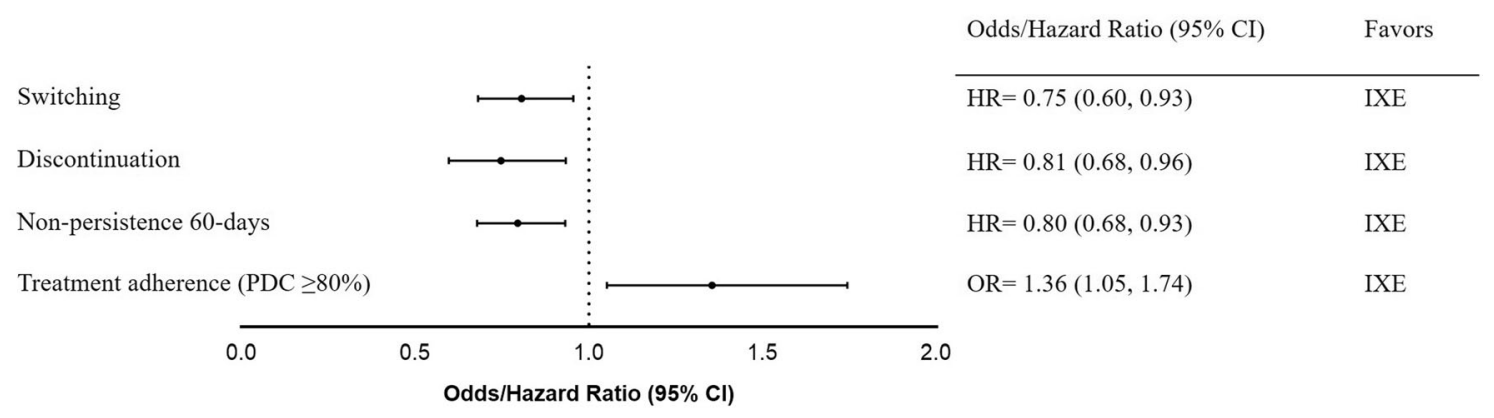

Fig. 3 Multivariable adjusted analyses of treatment patterns over 18-month follow-up period, patients with psoriasis receiving ixekizumab or secukinumab. CI

compared with SEC users (HR 0.75, 95\% CI 0.60-0.93) (Fig. 3).

\section{DISCUSSION}

Real-world studies comparing treatment patterns of IXE versus SEC among biologic-experienced patients with psoriasis are limited. This retrospective study used administrative claims databases to compare real-world treatment patterns of biologic-experienced patients with psoriasis using IXE or SEC over an 18-month follow-up period. Biologic-experienced IXE users had better persistence and high adherence compared to biologic-experienced SEC users; IXE users also had lower discontinuation and switching rates compared to SEC users. In the multivariate analyses, IXE demonstrated lower risks of non-persistence, discontinuation, and switching and a higher likelihood of high adherence compared with SEC.

A prior study by our group comparing treatment patterns between IXE and SEC users in a mixed population of biologic-naïve and biologic-experienced patients demonstrated relatively similar overall results despite having a variable and shorter length of follow-up (IXE 14 vs. SEC 16 months) [16]. Both groups had a similar proportion of biologic-experienced patients with psoriasis (IXE 66\% and SEC 68\%). IXE users had a lower risk of non-persistence (HR 0.82, 95\% CI 0.71-0.95) and discontinuation (HR 0.82, 95\% CI, 0.70-0.96), and a higher likelihood of high adherence (OR 1.32; 95\% CI 1.07-1.63) compared with SEC users. However, confidence interval, HR hazard ratio, IXE ixekizumab, OR odds ratio, PDC proportion of days covered

our prior study found no differences in the risk of switching to a different biologic or non-biologic treatment between IXE and SEC [16], whereas the present study showed that IXE users had a significantly lower risk of switching to a different biologic than SEC users.

Other studies have examined discontinuation rates among biologic-experienced IXE or SEC users. Murage et al. reported a 19.1\% discontinuation rate using 60 days allowable gap and a $12.6 \%$ discontinuation rate using 90 days allowable gap for biologic-experienced IXE users over a median follow-up of 201 days [18]. In studies reported by van den Reek et al. and Georgakopoulos et al., discontinuation rates for SEC users were 25\% (during 18-month followup) and $27 \%$ (during 12-month follow-up), respectively $[19,20]$. Most of the patients in the study reported by Georgakopoulos et al. (100\%) and van den Reek et al. (83\%) were biologicexperienced. Differences in discontinuation rates between previous reports and the current study may be due to differences in patient population, e.g., the Georgakopoulos et al. study included patients with psoriasis activity severity index (PASI) score $\geq 10$ at baseline [20] and the van den Reek et al. study was restricted to patients with no prior SEC exposure $[19,21]$. Study sample sizes (van den Reek et al., $n=196$, Georgakopoulos et al., $n=41$; current study, $n=780$ ) and length of follow-up may have contributed to the different outcomes as well.

In the absence of clinical measures, realworld treatment patterns data can serve as a good proxy for clinical outcomes [22]. Curtis 
et al. validated the use of administrative claims data to assess clinical effectiveness of medications for rheumatoid arthritis [23]. The current findings of favorable treatment patterns among biologic-experienced IXE users compared to SEC users are consistent with findings from clinical trials and other real-world studies examining clinical outcomes [24-26]. A real-world study comparing clinical outcomes between IXE and SEC patients with psoriasis who have two or more previous biologic therapies demonstrated a significantly higher PASI 75 (75\% improvement in PASI) response among IXE-treated than SEC-treated patients at week 52 (IXE 100\% vs. SEC 54.5\%; $p=0.039$ ) [25]. Likewise, indirect comparison using network meta-analysis showed that IXE users had higher PASI 90 response rate than SEC (72.1\% vs. $67.0 \%)$ during 48-52 weeks of treatment [26]. Together, these findings support a favorable effectiveness profile of IXE in the routine care of biologicexperienced patients with psoriasis as compared with SEC. This evidence may assist practitioners as they consider treatment options within the IL-17 biologic class for patients with psoriasis who have previous biologic experience.

There are a number of limitations to these data. This analysis was conducted using claims data, which are subjected to data coding limitations and data entry errors. Prior biologic exposure was based on a 6-month pre-period and therefore may have missed patients who were treated earlier than the pre-index period. Treatment pattern analysis assumed patients took medications as prescribed but we did not verify the patients actually took the medications. Residual differences not captured in claims may have introduced unobserved imbalances. Since selected databases were limited to patients with commercial health coverage or private Medicare supplemental coverage, results of this analysis may not be generalizable to patients with psoriasis with other insurance plans or without health insurance coverage.

\section{CONCLUSION}

IXE users exhibited higher persistence and adherence with a lower risk of discontinuation and switching compared with SEC users over 18 months of follow-up among biologic-experienced patients with psoriasis. These results add to existing data by providing comparative evidence that biologic-experienced patients treated with IXE are more likely to remain on treatment longer and use medication more persistently compared to patients treated with SEC. These findings generated from real-world clinical settings could aid healthcare providers in making treatment decisions for biologic-experienced patients with psoriasis.

\section{ACKNOWLEDGEMENTS}

Funding. This study and the journals rapid service fee was sponsored by Eli Lilly and Company, Indianapolis, USA.

Authorship. All named authors meet the International Committee of Medical Journal Editors (ICMJE) criteria for authorship for this article, take responsibility for the integrity of the work as a whole, and have given their approval for this version to be published.

Author Contributions. NS had full access to all the data in the study and take responsibility for the integrity of the data and the accuracy of the data analysis. Concept and design: $\mathrm{AB}, \mathrm{NS}$, $\mathrm{RB}, \mathrm{BZ}$, and MJM. Acquisition, analysis, or interpretation of data: $\mathrm{AB}, \mathrm{NS}, \mathrm{RB}, \mathrm{NS}, \mathrm{TLR}, \mathrm{BZ}$, BA, CRL, NMZ, MJM. All authors contributed to data analyses, drafting, and revising the manuscript.

Medical Writing, Editorial, and Other Assistance. The authors thank Scott A Kern (Eli Lilly and Company, Indianapolis, Indiana, USA) for providing inputs during study design and implementation. Rahul Nikam, an employee of Eli Lilly Services India Pvt. Ltd. provided writing support.

Disclosures. Independent analyses were conducted by IBM Watson Health. Eli Lilly and Company and IBM Watson Health collaborated on designing the study and interpreting results. 
Andrew Blauvelt, scientific adviser/clinical study investigator: AbbVie, Abcentra, Aligos, Almirall, Amgen, Arcutis, Arena, Aslan, Athenex, Boehringer Ingelheim, Bristol-Myers Squibb, Dermavant, Eli Lilly and Company, Evommune, Forte, Galderma, Incyte, Janssen, Landos, Leo, Novartis, Pfizer, Rapt, Regeneron, Sanofi Genzyme, Sun Pharma, and UCB Pharma. Nianwen Shi, Carolyn R. Lew, and Nicole M. Zimmerman are employed by IBM Watson Health, which was compensated by Eli Lilly and Company for completing this research. Russel Burge, Najwa Somani, Terri L. Ridenour, Baojin Zhu, Bilal Atiya, and Mwangi J. Murage are full-time employees and/or stockholders of Eli Lilly and Company.

Compliance with Ethics Guidelines. All study data were accessed with protocol compliant with the US patient confidentiality requirements, including the Health Insurance Portability and Accountability Act (HIPAA) of 1996 regulations. As all databases used in the study are fully de-identified and compliant with the HIPAA, this study was exempt from institutional review board approval.

Data Availability. Data for these analyses come from a proprietary database, the IBM MarketScan ${ }^{\circledR}$ Research Databases and are only available through third-party license. As such, the authors cannot make these data publicly available due to data use agreements. Other researchers can access these data by purchasing a license through IBM Watson Health. Interested individuals may see https://www.ibm. com/products/marketscan-research-databases for more information on accessing the MarketScan ${ }^{\circledR}$ Research Databases.

Open Access. This article is licensed under a Creative Commons Attribution-NonCommercial 4.0 International License, which permits any non-commercial use, sharing, adaptation, distribution and reproduction in any medium or format, as long as you give appropriate credit to the original author(s) and the source, provide a link to the Creative Commons licence, and indicate if changes were made. The images or other third party material in this article are included in the article's Creative Commons licence, unless indicated otherwise in a credit line to the material. If material is not included in the article's Creative Commons licence and your intended use is not permitted by statutory regulation or exceeds the permitted use, you will need to obtain permission directly from the copyright holder. To view a copy of this licence, visit http://creativecommons.org/licenses/by$\mathrm{nc} / 4.0 /$.

\section{REFERENCES}

1. Leonardi C, Reich K, Foley P, et al. Efficacy and safety of ixekizumab through 5 years in moderateto-severe psoriasis: long-term results from the UNCOVER-1 and UNCOVER-2 phase-3 randomized controlled trials. Dermatol Ther (Heidelb). 2020;10: 431-47.

2. Blauvelt A, Papp KA, Griffiths CE, et al. Efficacy and safety of guselkumab, an anti-interleukin-23 monoclonal antibody, compared with adalimumab for the continuous treatment of patients with moderate to severe psoriasis: results from the phase III, double-blinded, placebo- and active comparatorcontrolled VOYAGE 1 trial. J Am Acad Dermatol. 2017;76:405-17.

3. Thaçi D, Körber A, von Kiedrowski R, et al. Secukinumab is effective in treatment of moderate-tosevere plaque psoriasis: real-life effectiveness and safety from the PROSPECT study. J Eur Acad Dermatol Venereol. 2020;34:310-8.

4. Diotallevi F, Campanati A, Radi G, Molinelli E, Offidani A. Ixekizumab for treatment of moderate to severe plaque psoriasis: real world clinical experience. G Ital Dermatol Venereol. 2020;155:739-43.

5. Sbidian E, Chaimani A, Garcia-Doval I, et al. Systemic pharmacological treatments for chronic plaque psoriasis: a network meta-analysis. Cochrane Database Syst Rev. 2017;12:CD011535.

6. Langley RG, Elewski BE, Lebwohl M, et al. Secukinumab in plaque psoriasis-results of two phase 3 trials. N Engl J Med. 2014;371:326-38.

7. Menter A, Strober BE, Kaplan DH, et al. Joint AADNPF guidelines of care for the management and treatment of psoriasis with biologics. J Am Acad Dermatol. 2019;80:1029-72.

8. Murage MJ, Anderson A, Casso D, et al. Treatment patterns, adherence, and persistence among 
psoriasis patients treated with biologics in a realworld setting, overall and by disease severity. J Dermatolog Treat. 2019;30:141-9.

9. Doshi JA, Takeshita J, Pinto L, et al. Biologic therapy adherence, discontinuation, switching, and restarting among patients with psoriasis in the US Medicare population. J Am Acad Dermatol. 2016;74:1057-65.e4.

10. Warren RB, Smith CH, Yiu ZZN, et al. Differential drug survival of biologic therapies for the treatment of psoriasis: a prospective observational cohort study from the British Association of Dermatologists Biologic Interventions Register (BADBIR). J Invest Dermatol. 2015;135:2632-40.

11. Iskandar IYK, Warren RB, Lunt M, et al. Differential drug survival of second-line biologic therapies in patients with psoriasis: observational cohort study from the British Association of Dermatologists Biologic Interventions Register (BADBIR). J Invest Dermatol. 2018;138:775-84.

12. Menter A, Papp KA, Gooderham M, et al. Drug survival of biologic therapy in a large, disease-based registry of patients with psoriasis: results from the Psoriasis Longitudinal Assessment and Registry (PSOLAR). J Eur Acad Dermatol Venereol. 2016;30: 1148-58.

13. Wang TS, Tsai TF. Biologics switch in psoriasis. Immunotherapy. 2019;11:531-41.

14. Bissonnette R, Luger $\mathrm{T}$, Thaçi $\mathrm{D}$, et al. Secukinumab demonstrates high sustained efficacy and a favourable safety profile in patients with moderate-tosevere psoriasis through 5 years of treatment (SCULPTURE Extension Study). J Eur Acad Dermatol Venereol. 2018;32:1507-14.

15. Papp KA, Leonardi CL, Blauvelt A, et al. Ixekizumab treatment for psoriasis: integrated efficacy analysis of three double-blinded, controlled studies (UNCOVER-1, UNCOVER-2, UNCOVER-3). Br J Dermatol. 2018;178:674-81.

16. Blauvelt A, Shi N, Burge R, et al. Comparison of realworld treatment patterns among patients with psoriasis prescribed ixekizumab or secukinumab. J Am Acad Dermatol. 2020;82:927-35.

17. Berger ML, Sox H, Willke RJ, et al. Good practices for real-world data studies of treatment and/or comparative effectiveness: recommendations from the Joint ISPOR-ISPE Special Task Force on realworld evidence in health care decision making. Value Health. 2017;20:1003-8.

18. Murage MJ, Gilligan AM, Tran O, et al. Ixekizumab treatment patterns and healthcare utilization and costs for patients with psoriasis. J Dermatolog Treat. 2021;32:56-63.

19. van den Reek JMPA, van Vugt LJ, van Doorn MBA, et al. Initial results of secukinumab drug survival in patients with psoriasis: a multicentre daily practice cohort study. Acta Derm Venereol. 2018;98:648-54.

20. Georgakopoulos JR, Ighani A, Phung M, Yeung J. Drug survival of secukinumab in real-world plaque psoriasis patients: a 52-week, multicenter, retrospective study. J Am Acad Dermatol. 2018;78: 1019-20.

21. Egeberg A, Ottosen MB, Gniadecki R, et al. Safety, efficacy and drug survival of biologics and biosimilars for moderate-to-severe plaque psoriasis. $\mathrm{Br} \mathrm{J}$ Dermatol. 2018;178:509-19.

22. Thorneloe RJ, Bundy C, Griffiths CE, Ashcroft DM, Cordingley L. Adherence to medication in patients with psoriasis: a systematic literature review. Br J Dermatol. 2013;168:20-31.

23. Curtis JR, Baddley JW, Yang S, et al. Derivation and preliminary validation of an administrative claimsbased algorithm for the effectiveness of medications for rheumatoid arthritis. Arthritis Res Ther. 2011;13:R155-R.

24. Blauvelt A, Papp KA, Sofen $\mathrm{H}$, et al. Continuous dosing versus interrupted therapy with ixekizumab: an integrated analysis of two phase 3 trials in psoriasis. J Eur Acad Dermatol Venereol. 2017;31: 1004-13.

25. Herrera-Acosta E, Garriga-Martina GG, Suárez-Pérez JA, Martínez-García EA, Herrera-Ceballos E. Comparative study of the efficacy and safety of secukinumab vs ixekizumab in moderate-to-severe psoriasis after 1 year of treatment: real-world practice. Dermatol Ther. 2020;33:e13313.

26. Armstrong AW, Soliman AM, Betts KA, et al. Comparative efficacy and relative ranking of biologics and oral therapies for moderate-to-severe plaque psoriasis: a network meta-analysis. Dermatol Ther (Heidelb). 2021;11:885-905. 